\title{
FT-Raman spectroscopy study for skin cancer diagnosis
}

\author{
Lilian de Oliveira Nunes ${ }^{\mathrm{a}, *}$, Aírton Abrahão Martin ${ }^{\mathrm{a}}$, Landulfo Silveira Jr. ${ }^{\mathrm{b}}$ and \\ Marcelo Zampieri ${ }^{\mathrm{c}}$ \\ ${ }^{a}$ Laboratory of Biomedical Vibrational Spectroscopy, IP\&D - Institute of Research and Development, \\ UNIVAP - University of Vale do Paraíba, Av. Shishima Hifumi, 2911, São José dos Campos, SP, \\ ZIP 12244-000, Brazil \\ Tel.: +55 123947 1124; Fax: +55 123947 1149; E-mail: \{lili, amartin\}@univap.br \\ ${ }^{\mathrm{b}}$ Laboratory of Biomolecular Spectroscopy, IP\&D - Institute of Research and Development, \\ UNIVAP - University of Vale do Paraíba, Av. Shishima Hifumi, 2911, São José dos Campos, SP, \\ ZIP 12244-000, Brazil \\ ${ }^{\mathrm{c}}$ LIEC - Chemistry Department, UFSCar - Federal University of São Carlos, Rod. Washington Luíz, \\ km 235, Cx Postal 676, São Carlos, SP, ZIP 13565-905, Brazil
}

\begin{abstract}
Early detection of cancer is still a great challenge in clinical oncology. Recently, Raman spectroscopy has been used for skin lesion detection. FT-Raman spectroscopy is a modern analytical tool and its use for cancer diagnosis will lead to several advantages for the patient as, for example, real time and less invasive diagnosis. The primary objective of this work was to use FT-Raman spectroscopy to detect spectral changes between benign and malignant (basal cell carcinoma - BCC) skin tissues. Those spectral changes can provide important information about the biochemical alterations between these two types of tissues. We have analyzed by FT-Raman eight set of samples histopathologically diagnosed as BCC and made a comparison with five set of samples diagnosed as benign tissue. We have found that the main spectral differences between these samples were in the shift region of $1220-1300 \mathrm{~cm}^{-1}$ and $1640-1680 \mathrm{~cm}^{-1}$. The vibration bands in these regions correspond to the amide III and to the amide I vibrations, respectively. Principal components analysis applied over all 13 samples could identify tissue type with $100 \%$ of sensitivity and specificity.
\end{abstract}

Keywords: FT-Raman spectroscopy, skin cancer, diagnosis, principal components analysis

\section{Introduction}

The incidence and mortality rates of skin cancer have increased dramatically over the world during de last decade. In 2002 the estimation of skin cancer in the United States is of 111,900 new cases (excluding basal cell carcinoma, BCC and squamous cell carcinoma, SCC) [1]. The situation is not different in Brazil and, as shown in Fig. 1, the incidence of skin cancer corresponds to about 25\% (62,190 cases/year) of all diagnosed malignant tumors [2]. The high incidence of skin cancer in Brazil is probably related to the long exposure of workers to the sunlight in the countryside and also by the excessive exposure of people to sunlight for leisure. Recently, the Brazilian public health authorities have made a major campaign educating people to protect themselves from the sunlight. Although this approach will help to control and prevent the increasing of the incidence rate of skin cancer, the early detection should be the main goal to decrease the mortality rate. Nowadays, the skin cancer diagnostic is done by histopathological

\footnotetext{
${ }^{*}$ Corresponding author.
} 


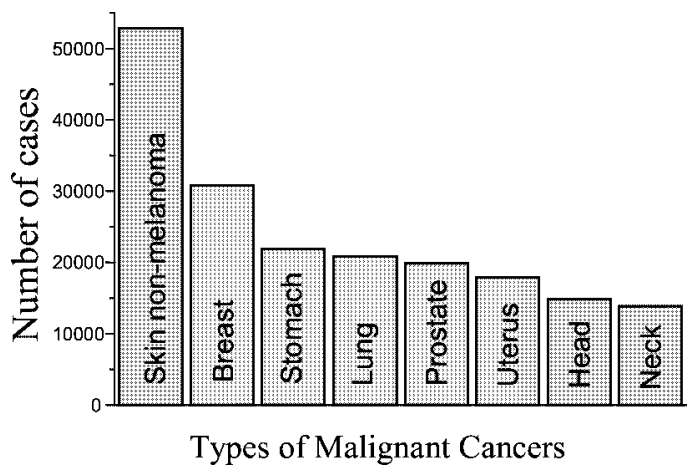

Fig. 1. Incidence and mortality numbers of main malignant neoplasms in Brazil [2].

biopsy where dermatologists count with experiences to the visual diagnosis of injuries in clinical routine examination by dermatoscopy technique. The evaluation of the injury characteristics is based on the so-called ABCDE system, i.e., Asymmetry, Border, Color, Dimension and Evolution of the lesion [3]. According to recent studies, up to $50 \%$ of early diagnosis of skin malignant lesions may escape detection during of clinical routine examinations, while experts achieve accuracy of approximately 80-90\% [4]. Many efforts have been made to develop techniques that can provide early diagnostic and prognostic procedure of tissues with better specificity.

All diseases lead to biochemical changes of cells and tissues. Therefore, the challenge of modern medicine is to find one analytical technique that investigates these changes using a non-invasive and nondestructive method that gives the diagnosis in real time. Few analytical methods satisfy this requirement and are sensible enough to study details of tissue compositions and structures. Among those methods, molecular vibrational spectroscopy is a potential technique to diagnose and study the evolution of human diseases, such as atherosclerosis [5] and precancerous and cancerous lesions [4,6-9].

Optical spectroscopy is able to detect biochemical changes of tissues that occur in inflammatory, benign or malignant pathologies of skin [10]. Malignant tissues have different optical properties, which are mainly caused by changes in the molecular structures of proteins and lipids in the tissues $[11,12]$. Those changes can be detected by vibrational spectroscopy and therefore, this technique has been considered a promising tool for the early cancer diagnosis. The Fourier Transform Raman spectroscopy (FT-Raman spectroscopy) is a powerful technique for biological analysis with an important advantage that lies in the decreased likelihood of sample fluorescence, typical of biological specimens [13]. The applicability of the Raman spectroscopy, in the long run, could be to complement the histopathological diagnosis, where the biopsy is unpracticable, for example, in multiple lesions or in incipient lesions that still not revealed clinically and also help removal of tumours by detecting security surgical limits. The use of Raman spectroscopy for cancer diagnosis has many advantages, for example, real time and less invasive procedures. It is a non-destructive technique that an optical fiber can be coupled for in vivo experiments, providing the capability for specific molecular fingerprinting and analysis [14].

Many studies have been performed as a tentative to correlate differences in the Raman spectrum to the tissue pathology [3-11]. FT-Raman have been used as a diagnostic in a variety of human diseases, mainly constructing a database and identifying main spectral features $[8,15]$. Skin cancer disease has been studied by FT-Raman and differences in the BCC and normal tissue spectra have been confirmed by neural network analysis [6].

Currently, a number of researchers employ multivariate statistics for spectral data analysis, interpretation and classification. Principal Components Analysis (PCA) is a very effective data reduction tech- 
nique. Basically it decomposes the spectra into factors, or principal components, which represent the most common variation to all the original data. Each principal component is related to the spectrum with a variable called score, representing the weight of that particular component to form the basis spectrum. These scores can be used to develop a model to classify the spectra into well-defined groups according to differences observed in the spectra [16], carried out by the scores of the first PCs.

In this work the objective is to detect vibrational changes in the FT-Raman spectra of benign and malignant tissues, identifying main features, and to correlate those changes with the histopathological analysis by using PCA.

\section{Materials and methods}

Skin tissues were obtained from oncologic routine biopsy and each piece was divided in two slices. One slice was sent to the histopathologist an the other one to the spectroscopic study. The patients were informed of the research by the Informing and Accordance Term, which was approved by the committee of ethics of UNIVAP. Samples were snap frozen and kept stored immersed in liquid nitrogen $\left(-196^{\circ} \mathrm{C}\right)$ in cryogenic bottles at the Laboratory of Biomedical Vibrational Spectroscopy, in the IP\&D, Institute of Research and Development, UNIVAP.

For collection of FT-Raman data, the samples were defrosted and warmed-up to room temperature washed with $0.9 \%$ physiological solution. The Raman spectrum collecting time was about 4 min and the total time for sample handling was about $10 \mathrm{~min}$, enough to prevent degradation and dehydration of the samples during analysis. We have used a FT-Raman spectrometer (RFS 100/S - Bruker Inc., Karlsruhe, Germany), with a Nd:YAG laser at $1064 \mathrm{~nm}$ as an excitation source. The output laser power was $300 \mathrm{~mW}$ and the data were obtained with 250 scans and resolution of about $4 \mathrm{~cm}^{-1}$.

Raman spectra were collected from eight sets of samples histopathologically diagnosed as basal cell carcinoma (BCC) and five sets of samples histopathologically diagnosed as benign tumor skin.

Raman spectra from each sample has been collected and stored by OPUS software (Bruker, Inc., Karlsruhe, Germany), where wavelength and intensity for most prominent peaks were evaluated and labeled. The OPUS data was converted to ASCII file prior Principal Components Analysis. The PCA routine was implemented using the MATLAB software (The Mathworks Inc., version 5.0), applied to all the obtained Raman spectra (a total of 13 ones) and the scores of the first PCs (principal components) were plotted in order to choose what PC can best differentiate the benign of the malignant tissue based on the histopathological results.

\section{Results}

Figure 2 shows typical FT-Raman spectra of benign and BCC skin tissues in the interval of 800 to $2300 \mathrm{~cm}^{-1}$. It can be observed differences in the overall intensity of the Raman spectra in both type of tissues, in which the BCC presents a lower intensity in the whole spectral range. The Raman spectral region of 1220 to $1300 \mathrm{~cm}^{-1}$ is assigned to the vibrational bands of the carbohydrate-amide III, the region of 1400 to $1500 \mathrm{~cm}^{-1}$ is assigned to proteins and lipids and the bands in the region between 1640 and $1680 \mathrm{~cm}^{-1}$ corresponds to the vibration of carbohydrate-amide I [6]. It can be seen that the main spectral differences between both benign and BCC tissues are in the shift region of amide I and amide III. The biochemical alterations of malignant tissue decreases significantly the intensity of the amide III vibration bands, in which band intensities are within two times less intense than the benign 


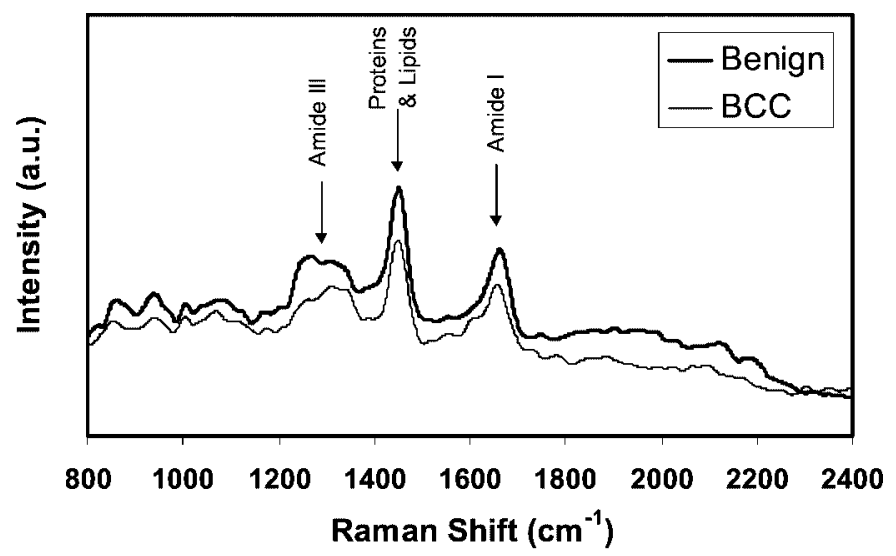

Fig. 2. FT-Raman spectrum of benign and BCC skin cancer. Vibrational bands occur in the following regions: Amide III: $1220-1300 \mathrm{~cm}^{-1}$, amide I: $1640-1680 \mathrm{~cm}^{-1}$, protein and lipid: $1400-1500 \mathrm{~cm}^{-1}$.
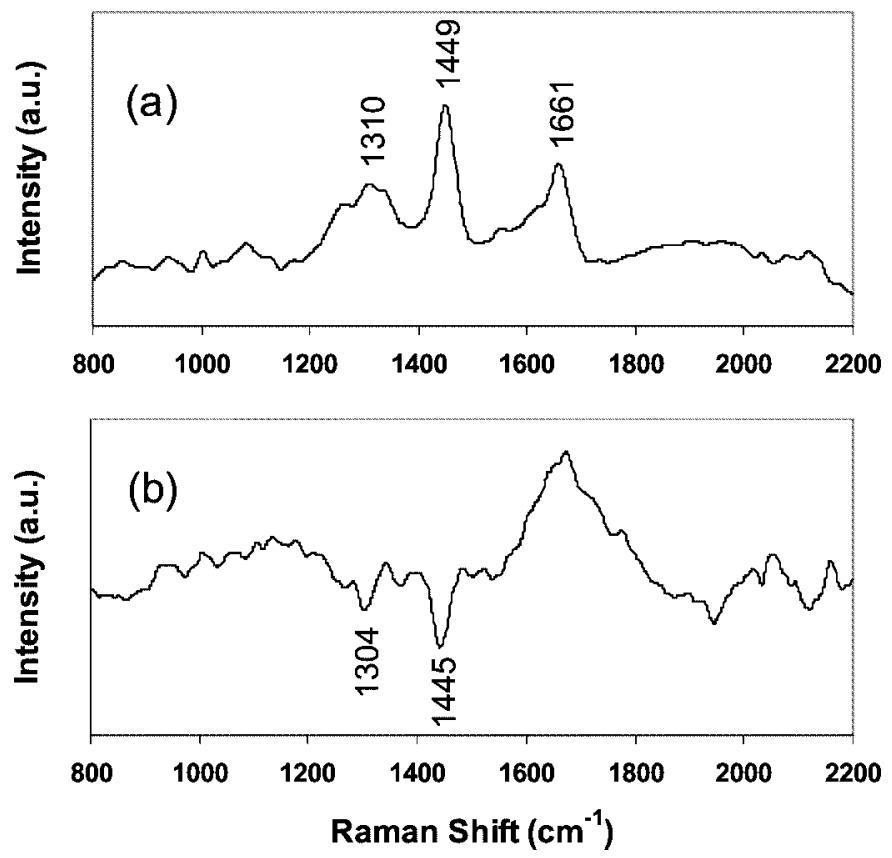

Fig. 3. (a) Principal component 1 and (b) principal component 2 obtained from the 13 Raman spectra.

samples. Also it is visible a reduction in the intensity of proteins and lipids peaks between 1440 and $1500 \mathrm{~cm}^{-1}$ of malignant tissue compared to benign tissue.

The principal components vectors resemble Raman spectra, but with positive as well as negative bands (valley shape). The first two PCs are shown in Fig. 3 which are responsible for about $90 \%$ of all spectral variations. Since the PC1 (Fig. 3a) has positive intensity bands and all the main bands are in the same positions as in the benign tissue spectrum, it gives a qualitative information about the tissue type. PC2 (Fig. 3b) has the same band positions, but some bands present the valley shape, indicating intensity differences for such bands. 


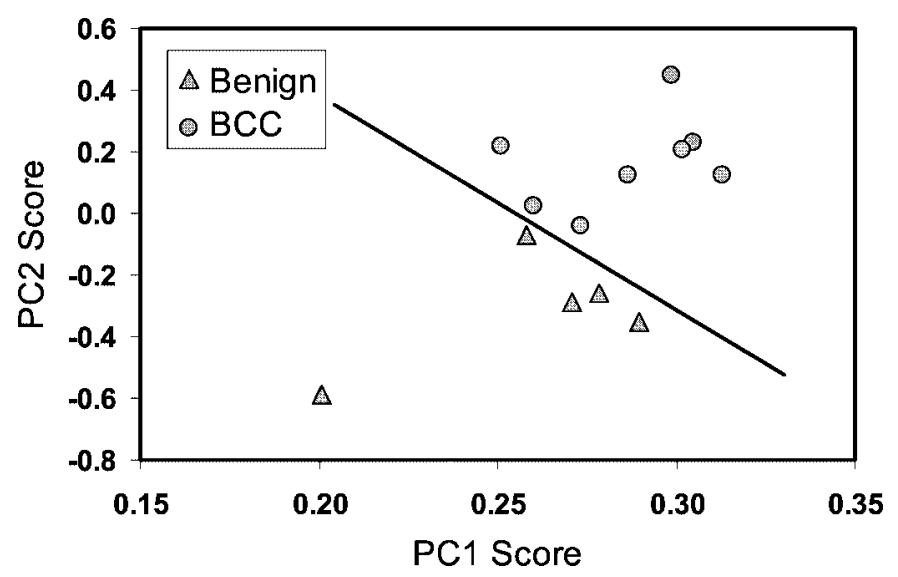

Fig. 4. Principal components scores 2 versus 1 (PC2 X PC1) for all the 13 FT-Raman spectra used in this study. The empirical diagnostic line drawn in this figure clearly separates benign from $\mathrm{BCC}$ tumor.

The first two principal components scores are plotted in Fig. 4. PC1 and PC2 scores are used to separate the data into the two tissue types according to the information found in each PC. In order to clearly separate the tissue types, an empirical diagnostic line based on the best visual separation was drawn. As can be seen in Fig. 4, this drawn line could correctly identify and separate the two types of tissues, giving $100 \%$ of sensitivity and specificity.

\section{Discussion and conclusion}

According to the studies made by Gniadecka et al. [6] and Fendel and Schrader [15], where they analyzed normal and malignant skin tissues it was observed spectral differences between the peaks of the amide I and III, proteins and lipids. We have found that these spectral differences also occur between malignant and benign skin tumor, and such differences can be used for optical diagnosis.

It has been demonstrated that it is possible to obtain reproducible high quality FT-Raman spectra of human skin tissues. The PCA can be effectively used for classification of human diseases, since each principal component has an unique spectral characteristic and the scores represent the magnitude of these characteristics correlated to the tissue histopathology. For the amount of spectra analyzed in this study the PCA could separate both tissue types with a $100 \%$ of agreement with histopathology. The principal components vectors could be used for prospective spectral analysis, by calculating the score of each principal component projected onto the prospective data, by means of a least square fitting routine, and plotting the first two PCs using the same diagnostic line.

Raman spectroscopy is one very promising technique for analysis of superficial cancer. By connecting optical fiber cables to collect light at the suspicious lesion in vivo, the technique make possible local diagnosis with results in a real time, nondestructively. The applicability of the Raman spectroscopy would be to assist the histopathological examination, mainly in situations which biopsy is not effective, for example, in multiples lesions or incipient lesions. 


\section{Acknowledgements}

Authors thank Prof. Dr. Edson Leite (UFSCar - Multidisciplinary Center for Development of Ceramic Materials) for the use of FT-Raman equipment, Dr. Carlos Oliveira Lopes, Dr. Sidney B. Cartaxo and Dr. Carlos Alberto Queiroz Carvalho for providing skin samples.

\section{References}

[1] American Cancer Society, in: Cancer Facts and Figures, American Cancer Society, New York, 2002, pp. 1-44.

[2] Ministério da Saúde, Coordination of programs of control of the cancer: "the problem of the cancer in Brazil", Instituto Nacional do Câncer (INCA), Rio de Janeiro, 2001.

[3] S. Tomatis, C. Bartoli, A. Bono, N. Cascinelli, C. Clemente and R. Marchesini, Spectrophotometric imaging of cutaneous pigmented lesions: discriminant analysis, optical properties and histological characteristics, J. Photochem. Photobiol. 42 (1998), 32-39.

[4] M. Gniadecka, C.W. Hans, O.F. Nielsen, H.C. Daniel and H. Jana, Molecular distinctive abnormalities in benign and malignant skin lesions: studies by Raman spectroscopy, Photochem. Photobiol. 66 (1997), 418-423.

[5] L. Silveira, Jr., S. Sathaiah, R.A. Zângaro, M.T.T. Pacheco, M.C. Chavantes and C.A.G. Pasqualucci, Correlation between near-infrared Raman spectroscopy and the histopathological analysis of atherosclerosis in human coronary arteries, Lasers in Surgery and Medicine 30 (2002), 290-297.

[6] M. Gniadecka, H.C. Wulf, N.N. Mortensen, O.F. Nielsen and D.H. Christensen, Diagnosis of basal cell carcinoma by Raman spectroscopy, J. Raman Spectr. 28 (1997), 125-129.

[7] A. Mahadevan-Jansen and R. Richards-Kortum, Raman spectroscopy for the detection of cancers and precancers, J. Biomed. Optics 1 (1996), 31-70.

[8] K. Yano, S. Ohoshima, Y. Gotou, K. Kumaido, T. Moriguchi and H. Katayama, Direct measurement of human lung cancerous and noncancerous tissues by Fourier transform infrared microscopy: can an infrared microscope be used as a clinical tool?, Analyt. Biochem. 287 (2000), 218-225.

[9] N. Hirosawa, Y. Sakamoto, H. Katayama, S. Tonooka and K. Yano, In vivo investigation of progressive alterations in rat mammary gland tumours by near-infrared spectroscopy, Analyt. Biochem. 305 (2002), 156-165.

[10] M. Gniadecka, Potential for high-frequency ultrasonography, nuclear magnetic resonance, and Raman spectroscopy for skin studies, Skin Res. Technol. 3 (1997), 139-146.

[11] C.A. Morton and R.M. Mackie, Clinical accuracy of the diagnosis of cutaneous malignant melanoma, Br. J. Dermatol. 138 (1998), 283-287.

[12] R. Manoharan, Y. Wang and M.S. Feld, Histochemical analysis of biological tissues using Raman spectroscopy, Spectrochim. Acta Part A 52 (1996), 215-249.

[13] T. Nicolet, Introduction to Raman Spectroscopy, Madison, USA, 2001.

[14] C.J. Lima, S. Sathaiah, L. Silveira, Jr., R.A. Zângaro and M.T.T. Pacheco, Development of catheters with low fiber background signals for Raman spectroscopic diagnosis applications, Artificial Organs 24 (2000), 231-234.

[15] S. Fendel and B. Schrader, Investigation of skin and skin lesions by NIR-FT-Raman spectroscopy, Fresen. J. Analyt. Chem. 360 (1998), 609-613.

[16] P. Geladi and B.R. Kowalski, Partial least square regression: a tutorial, Analyt. Chim. Acta 185 (1986), 1-17. 


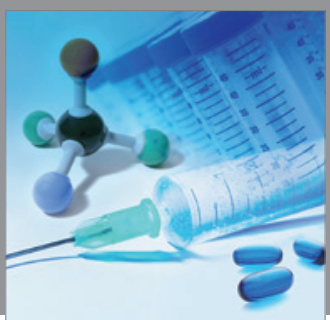

International Journal of

Medicinal Chemistry

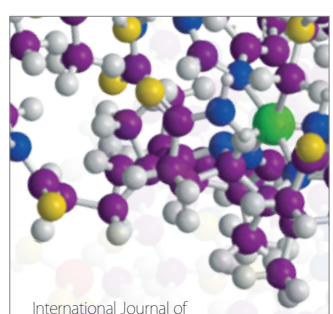

Carbohydrate Chemistry

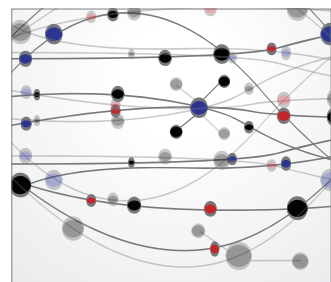

The Scientific World Journal
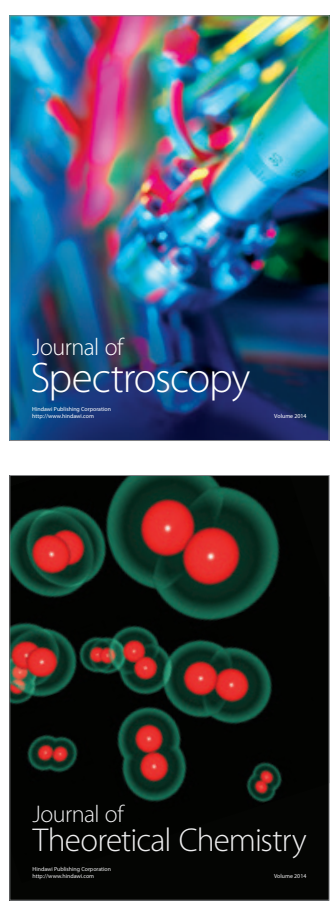
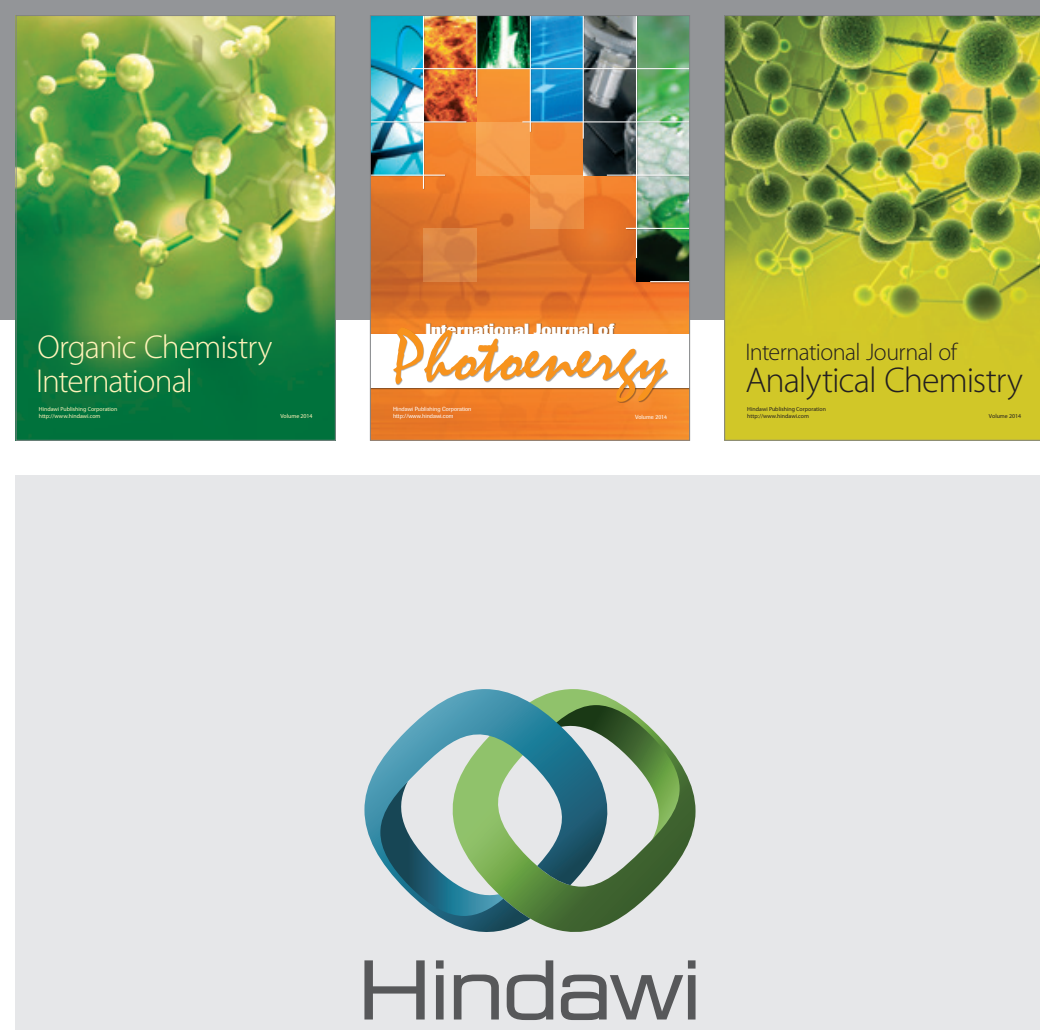

Submit your manuscripts at

http://www.hindawi.com
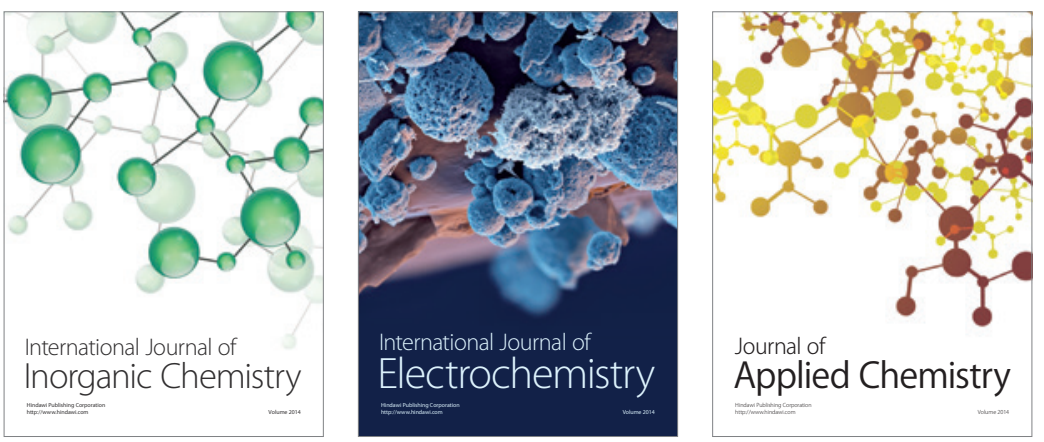

Journal of

Applied Chemistry
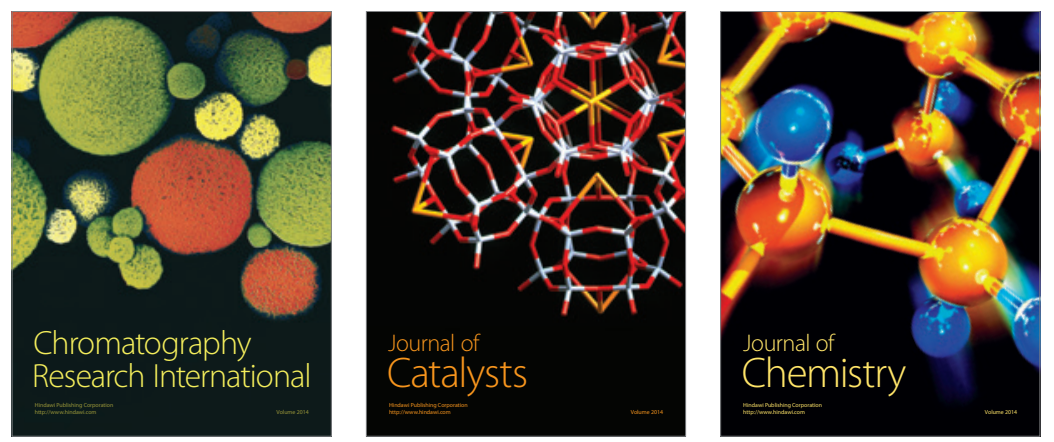
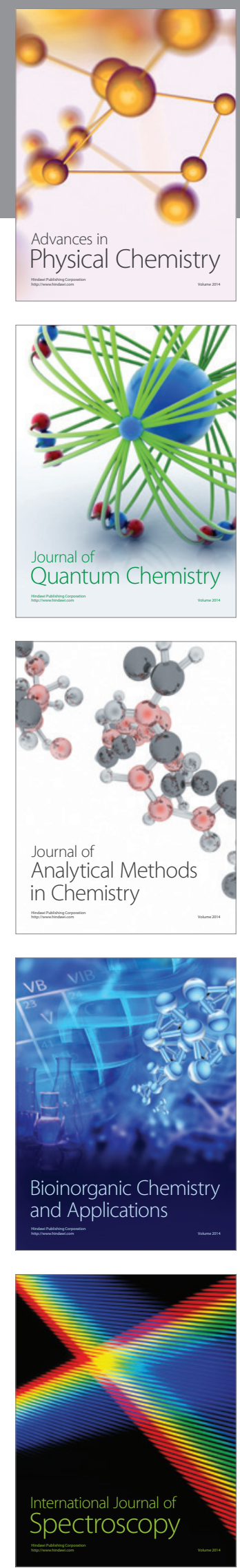